\section{G229(P) LONGITUDINAL AUDIT OF DIABETES CONTROL WITH INSULIN PUMP THERAPY OVER SEVEN YEARS OF TREATMENT - INTERIM RESULTS}

D Hawes, S Kanumakala. Paediatric Diabetes, Royal Alexandra Children's Hospital, Brighton, UK

\subsection{6/archdischild-2018-rcpch.224}

Aim Insulin pump therapy i.e. Continuous Subcutaneous Insulin Infusion therapy (CSII) is a well-recognised treatment modality in Type 1 Diabetes Mellitus (T1DM). NICE recommends a target $\% \mathrm{HbA} 1 \mathrm{c}$ level $\leq 6.5 \%$ to minimise long-term complication risk ${ }^{1}$. CSII can be considered in patients $<12$ years and in those whose $\% \mathrm{HbA} 1 \mathrm{c}$ have remained high $(\geq 8.5 \%)$ on multiple daily insulin therapy despite a high level of care. ${ }^{1}$

The aim of this audit is to review diabetes control over time in T1DM patients managed with CSII in our Paediatric Diabetes Unit (PDU).

Methods Retrospective review of diabetes control (\%HbA1c) of TIDM patients managed with CSII in our PDU (23/03/ 2009-10/01/2017).

Inclusion criteria $=$ All patients managed with CSII whose data is complete i.e. have a locally recorded pre-CSII\%HbA1c and are managed with CSII for at least one full year following switch to CSII.

Pre-CSII $\% \mathrm{HbA} 1 \mathrm{c}=$ mean of up to three $\% \mathrm{HbA} 1 \mathrm{c}$ recorded prior to switch to CSII.

Annual CSII\%HbA1c=mean of all $\% \mathrm{HbA} 1 \mathrm{c}$ recorded per whole year since switching to CSII.

Results In the time period reviewed there have been a total of 57 patients managed with CSII; seven patients were excluded from analysis.

There was a slight male preponderance $(1.08: 1,52 \%)$ with a mean age $( \pm S D)$ at diagnosis/transfer into our unit of 7.6 years $( \pm 4.5$ years $)$ and at switch to CSII of 10.2 years $( \pm 4.8$ years).

Analysis of data showed that those patients with the better control pre-switch generally maintained better control following switch to CSII.

Despite the fact that annual\%HbA1c showed that diabetes control generally worsens over time, cohort annual $\% \mathrm{HbA} 1 \mathrm{c}$ have remained relatively stable over the seven-year study period.

Conclusion Our PDU has a small number of patients managed with CSII. Results obtained were reflective of other studies which demonstrated worsening diabetes control over time ${ }^{2}$. Despite this, cohort annual results have remained relatively stable.

The difficulties of managing patients as they transition through puberty into adolescence/adulthood are well recognised. Supportive and educational opportunities must be maximised for optimal diabetes control and prevention of complications.

\section{G230(P) AUTOMATED BONE AGE ASSESSMENT YIELDS COMPARABLE RESULTS TO PAEDIATRIC RADIOLOGIST REPORTS}

${ }^{1} \mathrm{P}$ Jarvis, ${ }^{2} \mathrm{Y}$ Kumar, ${ }^{1} \mathrm{~K}$ Giles, ${ }^{1} \mathrm{~S}$ Thorogood. 'Radiology Department, Royal Cornwall Hospitals NHS Trust, Truro, UK; ${ }^{2}$ Child Health Department, Royal Cornwall Hospitals NHS Trust, Truro, UK

10.1136/archdischild-2018-rcpch.225
Aims To compare automated bone age assessment software with consultant paediatric radiologist reports in order to determine acceptability for use in our centre.

Background Bone age assessment is widely used in the evaluation of endocrine and genetic diseases as well as to assess the response to medical therapy. Our centre uses the Tanner and Whitehouse 2 radius, ulnar and short bones (TW2 RUS) method which involves grading these bones of the hand and wrist individually using maturity indicators from a reference book. This is then used to calculate a skeletal maturity score, which in turn is transformed into a bone age. This can be time consuming, especially for the less experienced reporter. It has also been shown that there is significant intra and interobserver variability in assessment. Automated methods have been developed to reduce reporting time as well as observer variation.

Methods Every bone age x-ray (hand and wrist) examination over a 3 month period was both reported by a consultant paediatric radiologist and assessed by an automated method (BoneXpert; Visiana, Denmark). We then analysed the TW2 results of each to see if the automated method would be acceptable for use in our centre. Results The mean variability between reporter and automated method was 0.71 years with a standard deviation of 0.58 years. This compares favourably with published studies which have separately reported TW2 specific interobserver variability of 0.74 years and an interobserver standard deviation of 0.71 years. Our results also equate to a $9.18 \%$ mean variability (SD 7.84) between reporter and automated software.

Conclusion Automated bone age assessment software yields comparable results to consultant paediatric radiologist reports. In particular the mean differences in bone age were similar to reported interobserver variability. This has led to our centre finding automated bone age software acceptable for use locally.

\section{G231(P) ABSTRACT WITHDRAWN}

\section{G232(P) RECOMBINANT HUMAN GROWTH HORMONE- SHOULD ACCESS GROW?}

D Burnside, V Puthi. Department of Paediatrics, Peterborough City Hospital, Peterborough, UK

\subsection{6/archdischild-2018-rcpch.226}

Aims NICE currently recommends the use of recombinant human growth hormone $(\mathrm{rHGH})$ to treat growth failure in 6 conditions based on a systematic review by Takeda et al. Growth hormone deficiency, Turner Syndrome, Prader-Willi syndrome, chronic renal insufficiency, SHOX deficiency, and children born small for gestational age with subsequent growth failure at over 4 years old.

Unfortunately, there are genetic syndromes in which short stature remains a feature, yet $\mathrm{rHGH}$ is not currently licensed. As a result, there are a small number of children who may benefit from $\mathrm{rHGH}$, yet remain unable to access it, with the subsequent theoretical risks of social isolation, educational underachievement and emotional distress associated with short stature. We hope these case reports can stimulate somewhat controversial discussions around the use of $\mathrm{rHGH}$ beyond its current mandate. 
Methods We present case reports from a UK hospital focussing on 2 patients with rare genetic conditions associated with short stature- Trichorhinophalangeal Syndrome and KBG Syndrome, accompanied by a summary of the current literature surrounding $\mathrm{rHGH}$ use in each condition.

Results There are 10 reported cases of rHGH use in TRPS, summarised below.

\begin{tabular}{lll}
\multicolumn{4}{l}{ Abstract G232(P) Table 1 } \\
\hline Paper & GH dose $(\mathrm{mg} / \mathrm{kg} /$ week) & Height standard deviation score change \\
\hline Naselli 1998 & 0.23 & 0 \\
& 0.23 & 0 \\
Stagi 2008 & 0.26 & +0.7 in 5 years \\
& 0.26 & +1.9 in 7 years \\
Sarafoglou 2010 & $0.3-0.43$ & +1.81 in 3 years \\
& $0.34-0.54$ & +1.95 in 2 years \\
Sohn 2012 & 0.2 & +0.4 in 10 years \\
& $?$ & 0 \\
Merjaneh 2014 & 0.28 & +1.0 in 2 years \\
Riedl 2004 & 0.2 & $?$ \\
\hline
\end{tabular}

Taken together, they suggest rHGH can be of benefit, and that earlier initiation of therapy is associated with better height outcomes. More information is needed before TRPS I can be considered a firm indication, but rHGH has potential to improve height outcomes in the short term.

Turning attention to KBG syndrome, there are only 2 detailled case reports of $\mathrm{GH}$ treatment in this condition (Reynart et al. 2015). These children increased their height by 0.6 and 1 SDS within 1 year of treatment, respectively.

Conclusion This work demonstrates the clinical features of 2 rare genetic conditions, and highlights the need for further debate around the potential of $\mathrm{rHGH}$ in maximising growth potential, with the ultimate aim of improving quality of life for patients with rare conditions including KBG syndrome and TRPS.

\section{REFERENCES}

1. Takeda A. Recombinant human growth hormone for the treatment of growth disorders in children: A systematic review and economic evaluation. Health Technol Assess 2010 September;14(42):1-209.

2. Naselli A. Trichorhinophalangeal syndrome type I in monozygotic twins discordant for hip pathology: Report on the morphological evolution of cone-shaped epiphyses and the unusual pattern of skeletal maturation. Pediatr Radiol 1998 November;28(11):851-5.

3. Stagi S. Partial growth hormone deficiency and changed bone quality and mass in type I trichorhinophalangeal syndrome. Am J Med Genet A 2008 June 15;146A (12):1598-604.

4. Sarafoglou K, Moassesfar S, Miller BS. Improved growth and bone mineral density in type I trichorhinophalangeal syndrome in response to growth hormone therapy. Clin Genet 2010 December;78(6):591-3.

5. Sohn YB. Clinical, biochemical, and genetic analysis of two Korean patients with trichorhinophalangeal syndrome type I and growth hormone deficiency. Ann Clin Lab Sci 2012 Summer;42(3):307-12.

6. Merjaneh L1. A novel TRPS1 gene mutation causing trichorhinophalangeal syndrome with growth hormone responsive short stature: A case report and review of the literature. Int J Pediatr Endocrinol 2014;2014(1):16.

7. Riedl S. Pronounced short stature in a girl with tricho-rhino-phalangeal syndrome II (TRPS II, Langer-Giedion syndrome) and growth hormone deficiency. Am J Med Genet Part A 2004;131:200-3.
8. Reynart N. Short stature in KBG syndrome: First responses to growth hormone treatment. Horm Res Paediatr 2015;83(5):361-4. doi:10.1159/000380908 [Epub: 2015 April 1]

\section{G233(P) CRANIOSYNOSTOSIS CAN OCCUR IN CHILDREN WITH NUTRITIONAL RICKET}

${ }^{1} \mathrm{~L}$ Forestier-Zhang, ${ }^{2} \mathrm{P}$ Arundel, ${ }^{1} \mathrm{R}$ Gilbey Cross, ${ }^{3} \mathrm{MZ}$ Mughal, ${ }^{2} \mathrm{AC}$ Offiah, ${ }^{1} \mathrm{MS}$ Cheung. ${ }^{1}$ Department of Paediatric Endocrinology, Evelina Children's Hospital, London, UK: ${ }^{2}$ Academic Unit of Child Health, The University of Sheffield, Sheffield, Sheffield, UK; ${ }^{3}$ Department of Paediatric Endocrinology, Royal Manchester Children's Hospital, Manchester, UK

\subsection{6/archdischild-2018-rcpch.227}

Background Severe vitamin D deficiency (VDD) is a common disorder which has complications including rickets, hypocalcaemia, hypotonia, delayed development and cardiomyopathy. Although nutritional rickets associated craniosynostosis has been reported, there is little awareness of this or knowledge about its clinical course or severity. We present five cases of late onset craniosynostosis in association with nutritional rickets.

Clinical presentation The diagnosis of craniosynostosis was made between the age of 16 months and 3 years $(n=5)$. All children had clinically evident scaphocephaly and radiological evidence of previous rickets. All children had risk factors for severe VDD: Afrocarribean or Asian ethnic backgrounds with darker skin pigmentation $(n=5)$; multiple food intolerances $(n=2)$ and prolonged breastfeeding with picky eating habits $(n=2)$. They presented in two ways:

Group $1(n=3)$ presented with clinical and radiological signs of severe rickets after a long period of untreated severe VDD. Serum $25 \mathrm{OH}$ vitamin D levels $<20 \mathrm{nmol} / \mathrm{L}$, elevated alkaline phosphatase, elevated parathyroid hormone (PTH) concentrations, low serum calcium and low phosphate concentrations. They were managed with treatment doses of vitamin D and calcium supplementation where necessary. In two patients, treatment had been completed and clinical signs resolved when the craniosynostosis was diagnosed.

Group $2(n=2)$ presented with sagittal suture ridging and scaphocephaly associated with resolving rickets on radiology. Clinically there were few other signs of VDD and serum $25 \mathrm{OH}$ vitamin $\mathrm{D}$ concentrations were $33-44 \mathrm{nmol} / \mathrm{L}$ with normal PTH and bone profiles.

CT in all cases showed fusion of the sagittal sutures. Three of the children also had multiple suture fusion. All in Group 1 were managed conservatively but Group 2 patients had raised intracranial pressure and both underwent surgical cranial vault remodelling.

Conclusions All the patients had nutritional rickets associated with craniosynostosis. Patients with late presentation and sagittal suture ridging went on to have emergency cranial vault remodelling. It is important to recognise this complication early and refer to the neurosurgeons and so prevent raised intracranial pressure. It is important to collect detailed data on this and study a larger cohort to raise awareness, establish the pathophysiology and try to prevent this complication. 